\title{
Zygomaticomaxillary suture maturation: A predictor of maxillary protraction? Part I - A classification method
}

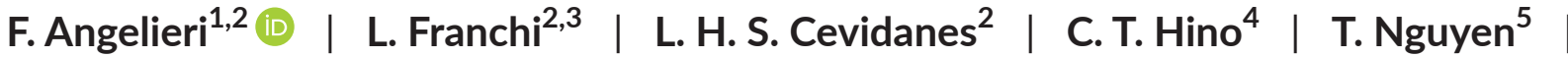 \\ J. A. McNamara Jr $6,7,8$
}

${ }^{1}$ Department of Orthodontics, Guarulhos University, Guarulhos, Brazil

${ }^{2}$ Department of Orthodontics and Pediatric Dentistry, School of Dentistry, The University of Michigan, Ann Arbor, MI, USA

${ }^{3}$ Department of Orthodontics, The University of Florence, Florence, Italy

${ }^{4}$ Special Studies in Orthodontics, São Paulo Military Hospital, São Paulo, Brazil

${ }^{5}$ Department of Orthodontics, School of Dentistry, University of North Carolina, Chapel Hill, NC, USA

${ }^{6}$ Department of Orthodontics and Pediatric Dentistry, School of Dentistry, Ann Arbor, MI, USA

${ }^{7}$ Cell and Development Biology, School of Medicine, Ann Arbor, MI, USA

${ }^{8}$ Center of Human Growth and Development, The University of Michigan, Ann Arbor, MI, USA

Correspondence

F. Angelieri, Programa de Pós-Graduação

em Odontologia, Praça Tereza Cristina, 229

Guarulhos, SP, Brazil.

Email: fernandaang@yahoo.com.br

\section{Structured Abstract}

Objective: The aim of this study was to present a method of classifying the maturational level of the zygomaticomaxillary sutures (ZMSs).

Methods: Cone-beam CT (CBCT) images from 74 subjects (5.6-58.4 years) were examined to define the radiographic stages of ZMS maturation. Five stages of maturation of the ZMS were identified and defined: Stage A-uniform high-density sutural line, with no or little interdigitation; Stage B-scalloped appearance of the high-density sutural line; Stage C-two parallel, scalloped, high-density lines, separated in some areas by small low-density spaces; Stage D-fusion in the inferior portion of the suture; and Stage E-complete fusion. Intra- and inter-examiner agreements were evaluated by weighted kappa tests.

Results: The intra- and inter-examiners reproducibility values demonstrated substantial to almost perfect agreement. No fusion of ZMSs was observed in patients up to 10 years of age. From 10 to 15 years, all maturational stages were identified. After 15 years of age, the majority of patients showed fusion of ZMSs.

Conclusions: The classification of ZMS maturation using CBCT is a reliable method that allows the assessment of the morphology of the ZMSs in the individual patient.

KEYWORDS

cone-beam computed tomography, cranial sutures, growth and development

\section{1 | INTRODUCTION}

Growth modification of the maxilla depends on the level of maturation of the circummaxillary sutures. ${ }^{1-5}$ The maxilla articulates with other facial bones by way of the transverse palatine suture, frontomaxillary sutures and ZMSs (Figure 1). In the posterior region, the palatine bone interposes between the maxilla (transverse palatine suture) and the pterygoid process of sphenoid bone (pterygopalatine suture). ${ }^{1}$ Interestingly, histological examination of human autopsy material demonstrated similar maturational stages of the transverse palatine and pterygopalatine sutures during the infantile, juvenile and adolescent growth stages. ${ }^{1}$ These sutures show an increasing interdigitation and complexity from the infantile through the adolescent stages, with complete fusion of the sutures in the adult. Clinically, the complexity of the interdigitations of the circummaxillary sutures or even their fusion can hamper desired orthopedic changes, such as those produced by maxillary protraction in patients with Class III malocclusion. ${ }^{6-11}$

The clinical parameter for the best timing for maxillary protraction using the face mask protocol is chronological age. However, different chronological ages have been proposed, with some authors recommending treatment up to $8,{ }^{12} 9,{ }^{13}$ and $10^{6,14}$ years of age or before puberty. ${ }^{7}$ On the other hand, other studies did not identify any differences in response according to chronological age..$^{2,15,16}$

Animal studies ${ }^{2,3,5}$ have demonstrated that the complexity of interdigitations is greater in the zygomaticomaxillary sutures than in other circummaxillary sutures. Kambara ${ }^{17}$ observed that the ZMSs 

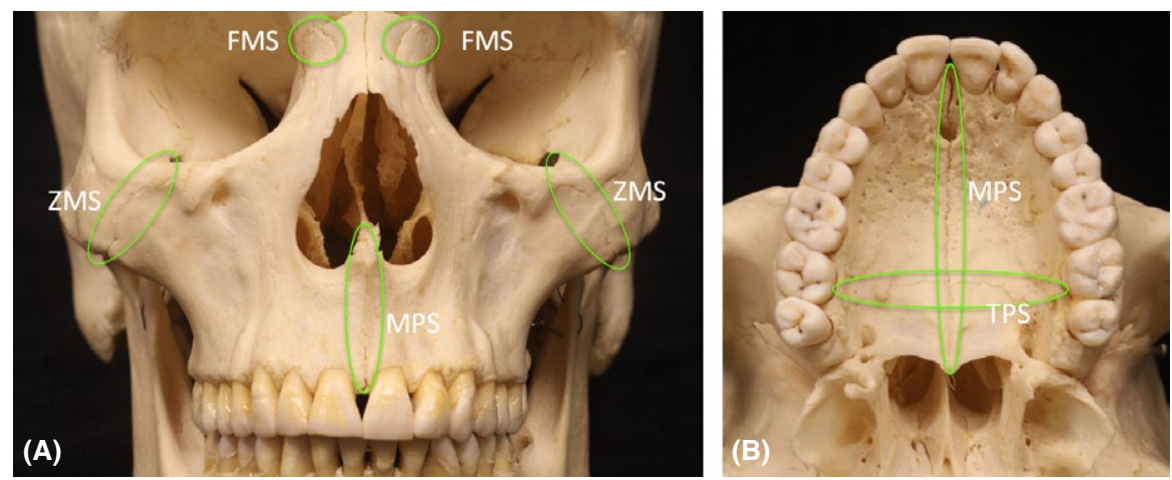

FIGURE 1 The maxillary sutures:

(A) Frontomaxillary sutures (FMS), zygomaticomaxillary sutures (ZMS), midpalatal suture (MPS). (B) Midpalatal and transverse (TPS) palatine sutures [Colour figure can be viewed at wileyonlinelibrary.com] presented similar histological findings, or even greater complexity of interdigitations, compared to other circummaxillary sutures in young (mixed dentition phase) and older (permanent dentition phase) monkeys. Studies that used finite-element analysis reported that the ZMSs showed a major resistance to orthopedic forces generated by rapid maxillary expansion (RME) and maxillary protraction. ${ }^{18,19}$ Furthermore, the ZMSs are the longest and thickest of these sutures, and they are oriented in the same direction as the force systems applied for maxillary protraction. ${ }^{4,5}$

One method of analysis of these sutures is by radiographic interpretation of cone-beam computed tomography (CBCT), a technique that provides 3D images of the oral and maxillofacial structures with relatively low cost, no image overlap, ease of accessibility and low radiation compared to multislice $\mathrm{CT}^{20}{ }^{20}$ Recently, Angelieri et al. ${ }^{21}$ described a method of classification of the midpalatal suture maturation on $\mathrm{CBCT}$ images that can be useful mainly for late adolescent and young adult patients in whom the efficacy of RME is unpredictable. This individual assessment of the maturation level of the circummaxillary sutures has the potential to provide a means of assessing the potential midfacial sutural response to maxillary protraction forces in Class III patients.

The aim of this study was to present a novel classification method for individual assessment of zygomaticomaxillary suture maturation as observed on СВCT images and to test the reliability of this new classification method.

\section{SUBJECTS AND METHODS}

Baseline diagnostic CBCT images acquired for clinical purposes from 74 subjects (50 females and 24 males; Table 1), with ages ranging from 5.6 to 58.4 years and with no history of previous orthodontic treatment, systemic diseases or syndromes, were examined to determine the radiographic stages of ZMS. This study was approved by the Institutional Review Board from the University of Michigan.

CBCT scan images were obtained using an ICAT $^{\text {TM }}$ Cone Beam 3-D Imaging System (Imaging Science International, Hatfield, PA, USA). Each subject was seated in an upright position with the Frankfort plane parallel to the floor during the scanning process. For all scans, the field of view (FOV) used was $16 \times 22-\mathrm{cm}$, and the scan time ranged from 20 to 40 seconds, with spatial resolution ranging from 0.25 to $0.30 \mathrm{~mm}$.
TABLE 1 Demographics of the sample regarding gender and age

\begin{tabular}{lllll} 
Gender & $5-<10 y$ & $10-<15 y$ & $>15 y$ & Total \\
\hline Female & 17 & 15 & 18 & 50 \\
Male & 03 & 14 & 07 & 24 \\
Total & 20 & 29 & 25 & 74 \\
\hline
\end{tabular}

\section{1 | Image analysis procedures}

The image analysis was performed using Invivo5 ${ }^{\mathrm{TM}}$ software (Anatomage, San Jose, CA, USA). The tortuousness of the $\mathrm{ZMS}^{22}$ requires the definition of a careful protocol to interpret cross-sectional images through the long axis of the suture. The following procedures were required to determine the maturational stages of the ZMS:

1. Head orientation: Using the reorientation module, head position was reoriented to correct for roll, pitch and/or yaw during image acquisition (Figure 2).

2. In sagittal view, the horizontal cursor (orange line) was placed at the tip of the nose parallel to the palatal plane (Figure 3). In this inferior-superior cross section determined sagittally, the axial view then displayed a portion of the oblique ZMSs bilaterally. The anteroposterior cursor (purple line) then was positioned transversely through the ZMSs bilaterally. These procedures allowed visualization of the ZMSs also in the coronal view (Figure 4).

For analysis of the tortuous path of the ZMSs, in the coronal view, the vertical cursor (green line) first was positioned along the ZMS on the left side (Figure 5C). These orientation steps allowed visualization of the suture in all views (sagittal, axial and coronal; Figure 5A-C). In the sagittal views, the inferior portion of the ZMS was more lateral and often overlapped the view of the cortical bone (Figure 6). For this reason, interpretation of the maturational stage of the inferior portion of the left ZMS required rotating the patient's head in a counterclockwise direction in the coronal view until the inferior portion of the ZMS was visualized appropriately in the sagittal view (Figure 7).

Radiographic interpretation required scrolling the sagittal cross sections to evaluate the medial and lateral path of the infraorbital portion of the ZMS. The same procedures were repeated for assessment of the ZMS on the right side and in the coronal view, so the patient's head was rotated in a clockwise direction (Figure 8). 
FIGURE 2 Use of head orientation tools to position the patient's head (roll, pitch and/or yaw) for better visualization of the ZMS after image acquisition [Colour figure can be viewed at wileyonlinelibrary.com]
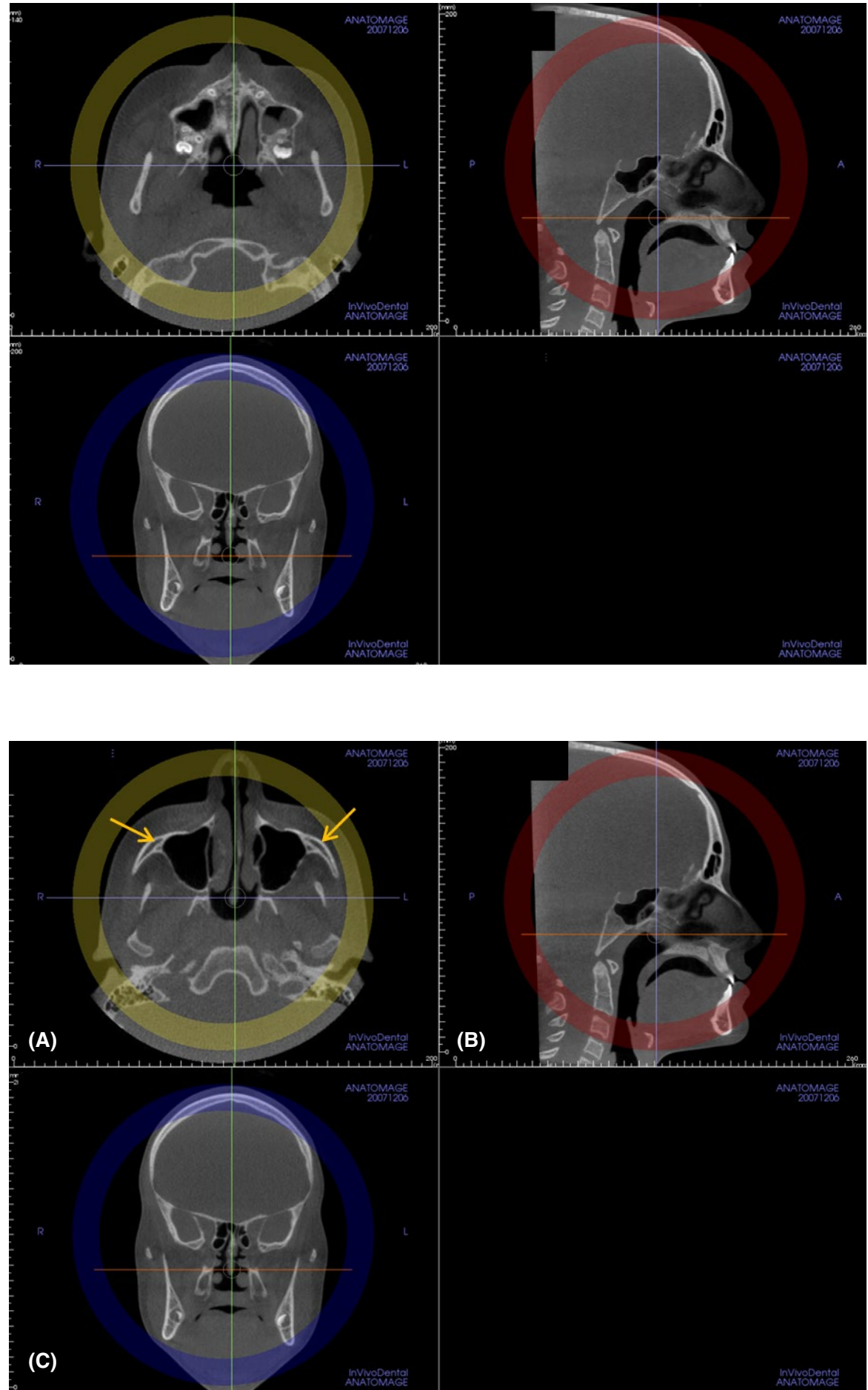

FIGURE 3 Visualization of the ZMS: in the sagittal view, the horizontal cursor (orange line) is placed at the tip of the nose parallel to the palatal plane (B). Note that in the axial view, the zygomaticomaxillary sutures are seen bilaterally (A) (arrows). The vertical cursor (green line) should be positioned on the midsagittal plane of the patient (C) [Colour figure can be viewed at wileyonlinelibrary.com] definition of each CBCT radiographic appearance of the sutural maturation stage followed the findings of previous histological ${ }^{2,4}$ and micro-CT studies ${ }^{23,24}$ on the morphological maturation of the ZMS. The following descriptive stages of ZMS maturation are proposed:

Stage A: The zygomaticomaxillary suture is a uniform high-density line (with no or little interdigitation), with decreased parasutural bone density. This suture has a tortuous path (Figure 9). 2,4,22,24,25 


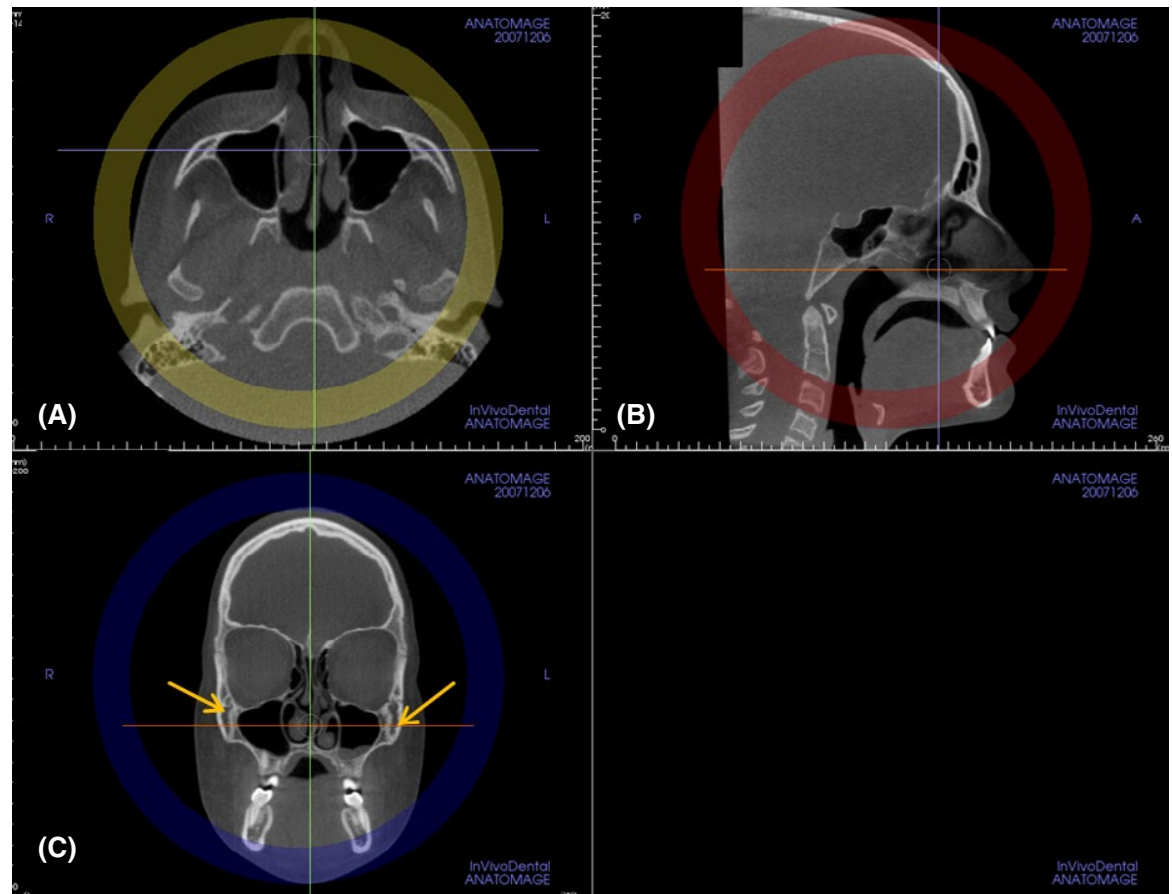

FIGURE 4 Visualization of the ZMS: in the axial view, the anteroposterior cursor (purple line) is positioned transversely through the ZMSs bilaterally (A). This approach allows the visualization of the ZMSs in the coronal view bilaterally (C) (arrows) [Colour figure can be viewed at wileyonlinelibrary.com]

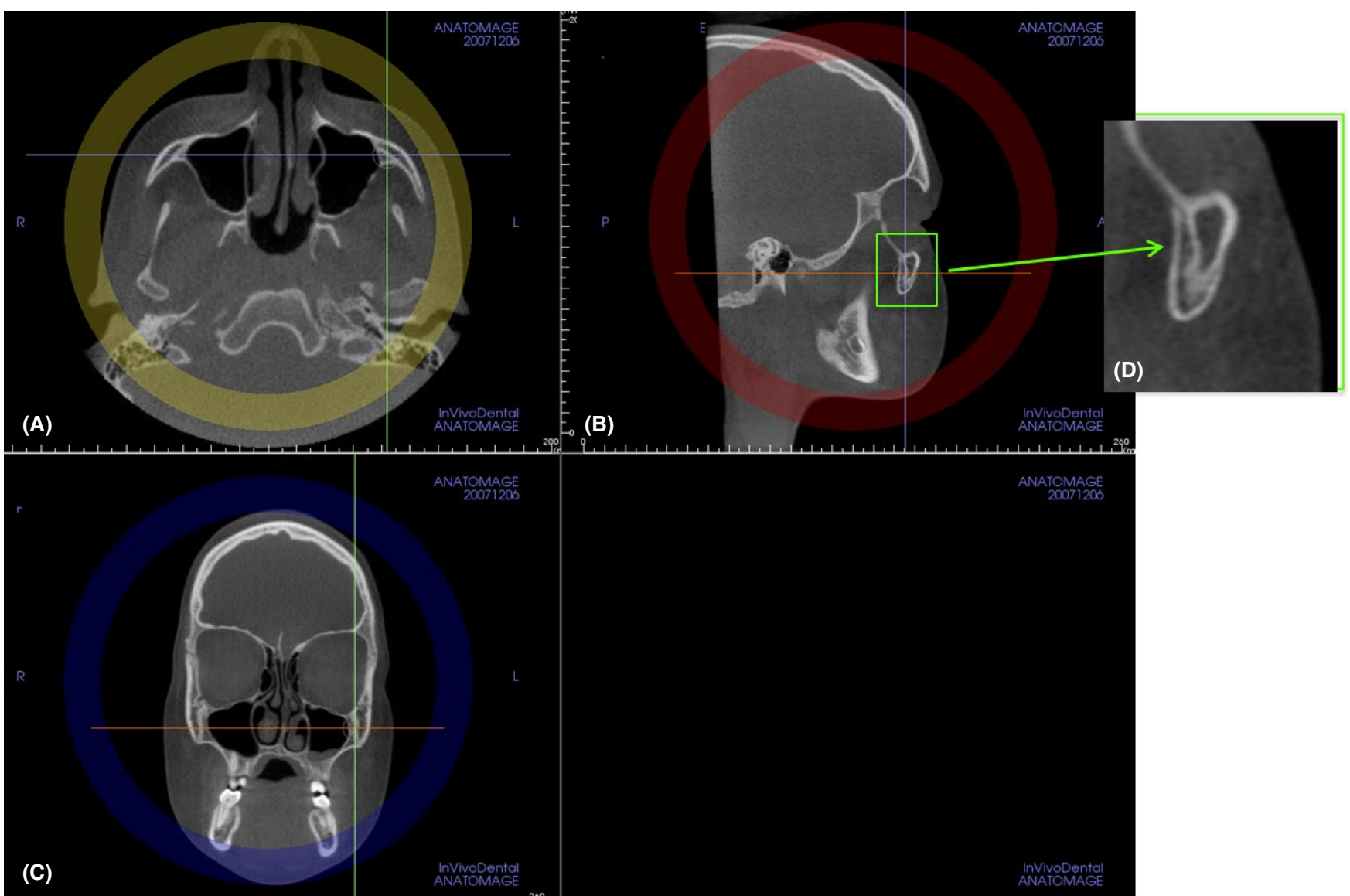

FIGURE 5 Visualization of the ZMS: in the coronal view (C), the vertical cursor (green line) then is positioned along the ZMS on the left side (yellow arrow) to allow clinicians to scroll along the suture and visualize its tortuous path and portions. The suture then is visualized in all multiplanar views (axial in A, sagittal in B and coronal in C views) (D) Close-up view of (B) sagittal view, where the maturation of the ZMS is identified (green arrow) [Colour figure can be viewed at wileyonlinelibrary.com] 
Stage B: ZMS can be seen as a thicker scalloped high-density line with some interdigitation. In a later phase, stage B also can be seen as a thicker scalloped high-density line in some areas, and in other areas

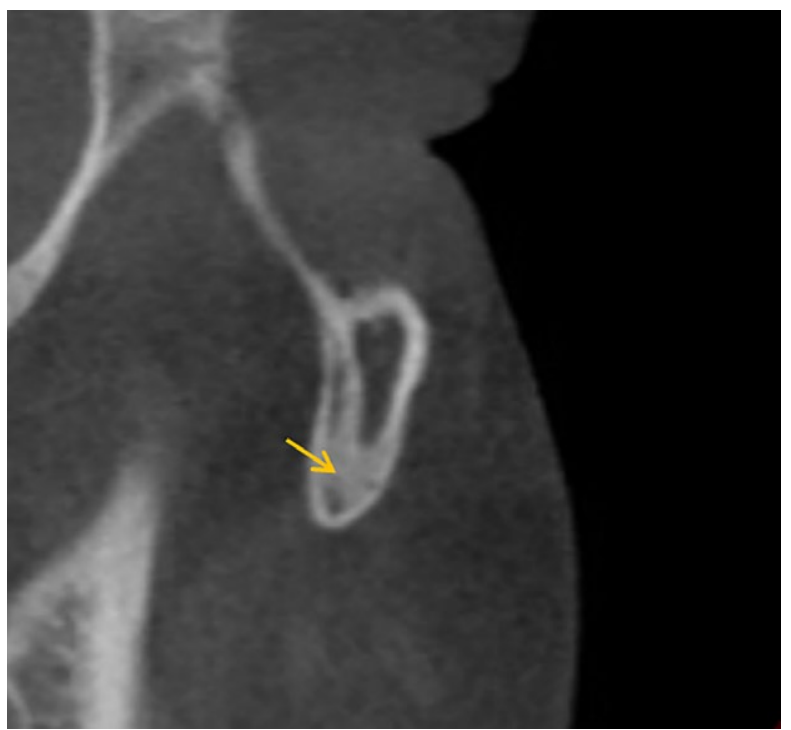

FIGURE 6 Sagittal view of the inferior portion of the ZMS. Note the inferior portion is more lateral and may overlap view of the cortical bone, with the false appearance of fusion (arrow) [Colour figure can be viewed at wileyonlinelibrary.com] as two thin, parallel, scalloped, high-density lines close to each other and separated by small low-density spaces (Figure 10). ${ }^{25,26}$ The parasutural bone density still is decreased.

Stage C: Stage C appears as two thin, parallel, scalloped, high-density lines that are close to each other and separated by small low-density spaces in the zygomatic and maxillary bones. The parasutural bone density remains decreased (Figure 11). ${ }^{25,26}$

Stage D: Fusion has occurred in a portion of the ZMS, usually in most inferior part of the suture, where the ZMS line cannot be visualized and the parasutural bone density is increased (high-density bone; Figure 12).

Stage E: The ZMS is not visible in many areas along the suture, i.e., there are many areas of fusion. ${ }^{13}$ The density of the parasutural bone is increased (Figure 13).

For patients who present wide maxillary sinus, the ZMSs can be located on the lateral walls of the maxillary sinus. In this case, although the ZMSs could appear fused, they are visible inferiorly and/or superiorly and laterally of the maxillary sinus (Figure 14). The ZMSs were analysed on right and left sides, and the more matured stage was considered.

All sagittal cross-sectional slices used for the assessment of the zygomaticomaxillary suture were selected and classified by the principal investigator (F.A.). These slices were arranged in a presentation (Microsoft Office PowerPoint 2007, Microsoft Corporation) with black
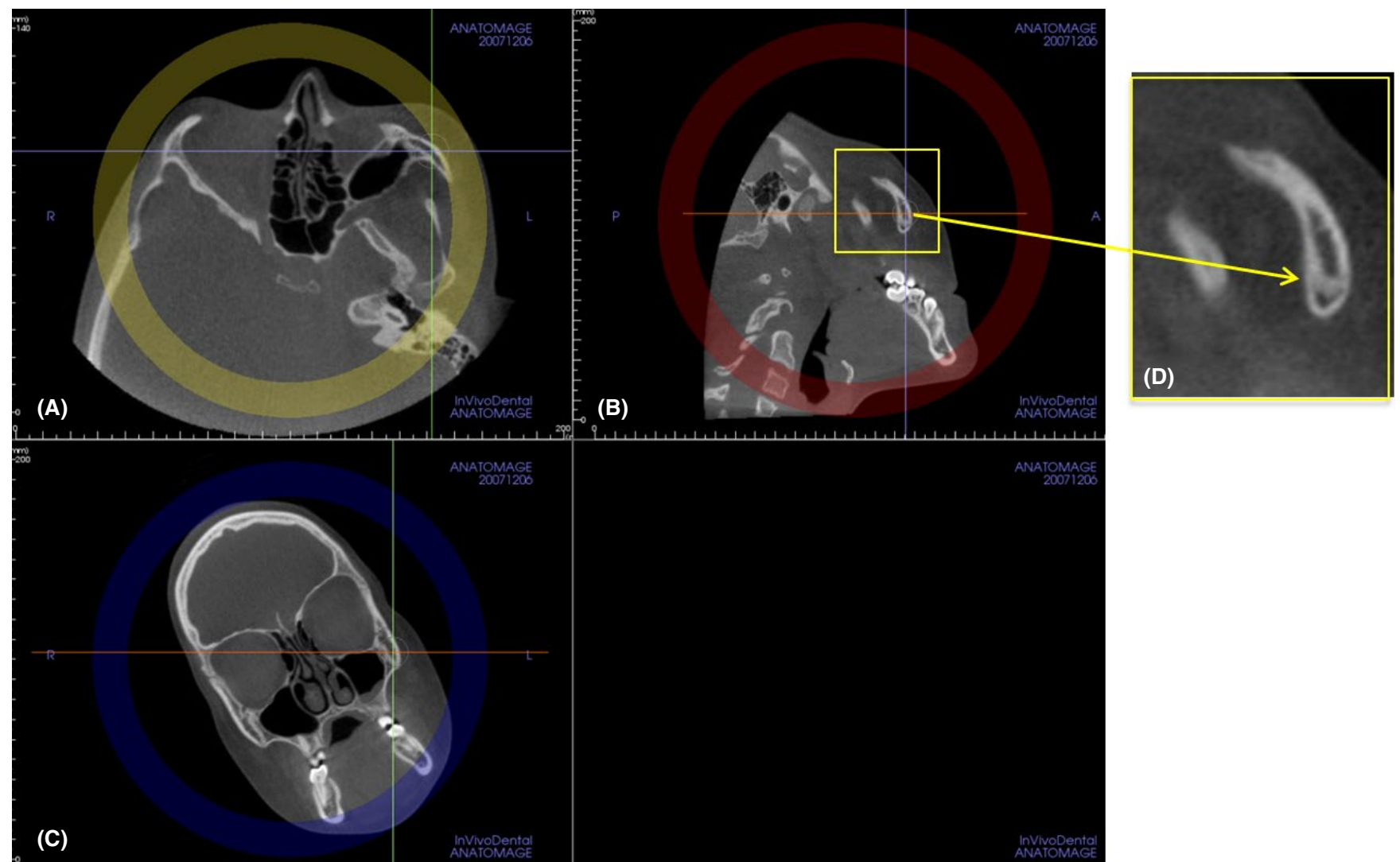

FIGURE 7 Proper radiographic interpretation of the maturational stage of the inferior portion of the ZMS requires rolling the patient's head in a counterclockwise direction in the coronal view (C) until the inferior portion of the ZMS is visualized properly in the sagittal view (B). (D) Close-up view of the inferior portion of the left ZMS [Colour figure can be viewed at wileyonlinelibrary.com] 


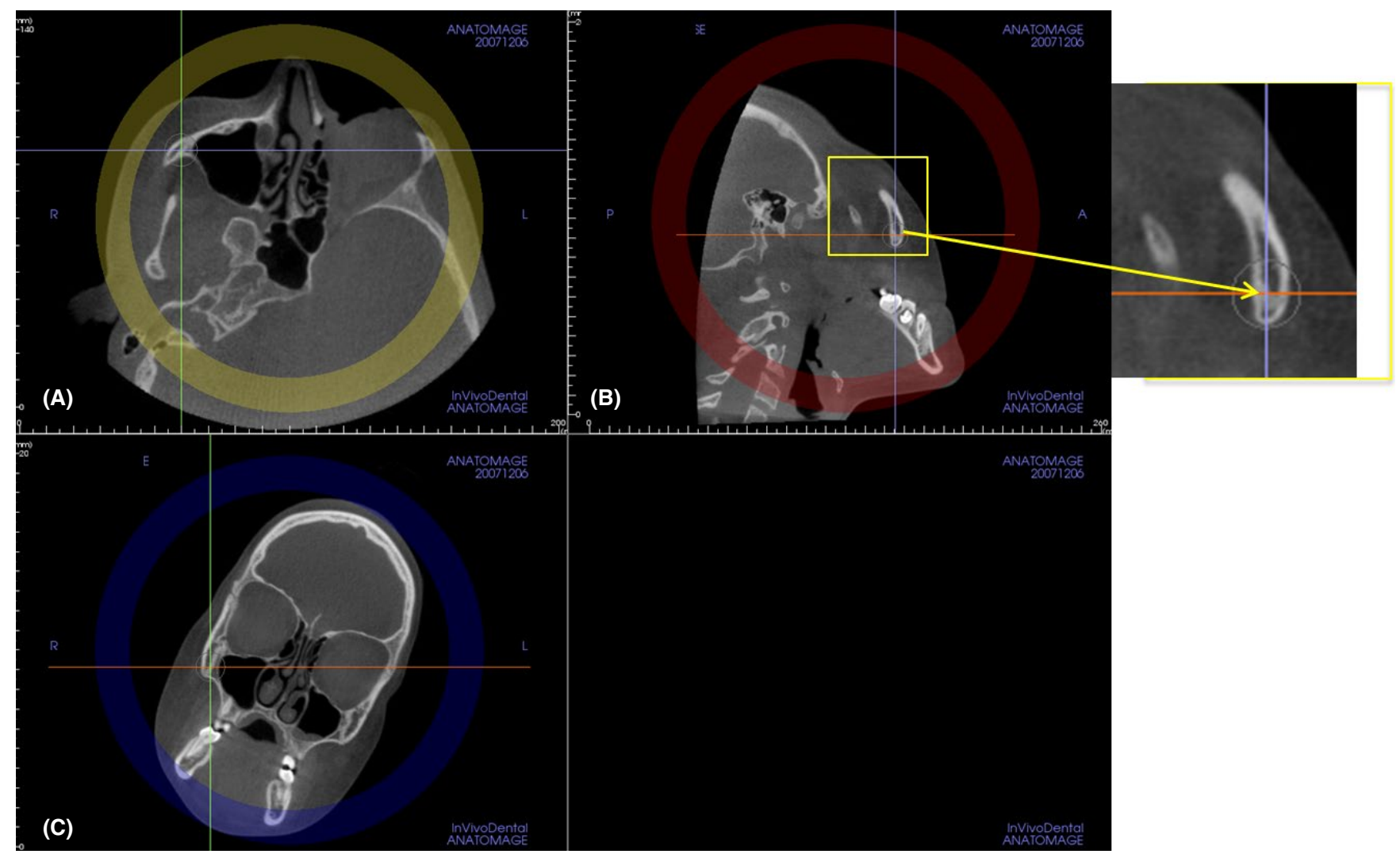

FIGURE 8 Proper radiographic interpretation of the maturational stage of the inferior portion of the right ZMS requires rolling the patient's head in a clockwise direction in the coronal view (C) until the inferior portion of the ZMS is visualized properly in the sagittal view (B). (D) Closeup view of the inferior portion of the right ZMS [Colour figure can be viewed at wileyonlinelibrary.com]

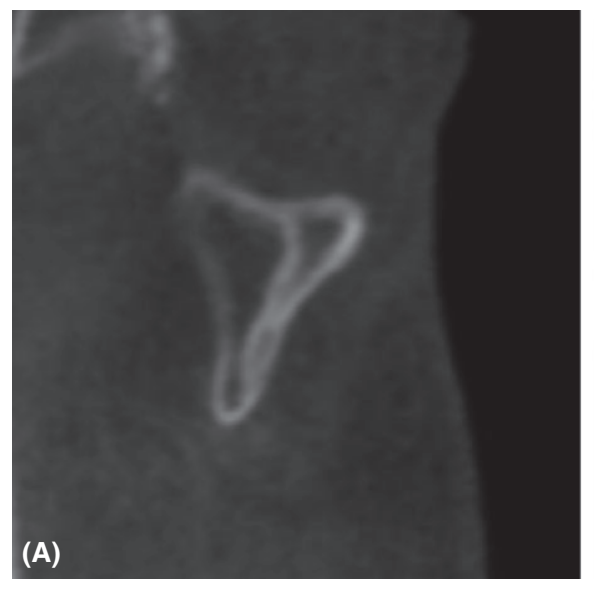

(B)

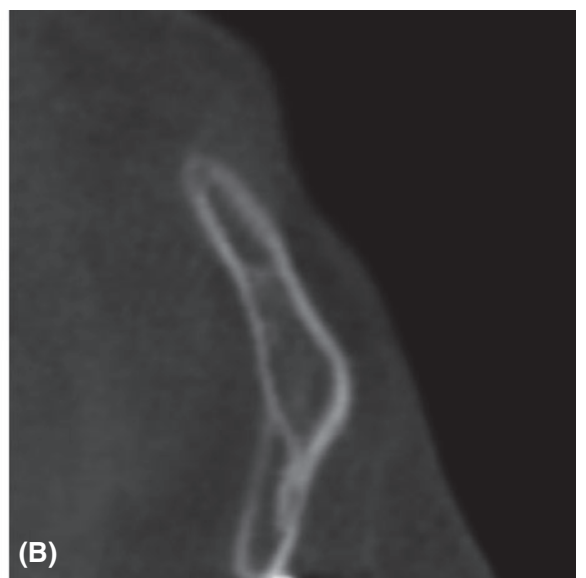

FIGURE 9 Stage A: the ZMS is a uniform high-density line (no or little interdigitation), with decreased parasutural bone density at infraorbital $(A)$ and infrazygomatic $(B)$ portions of this suture background and codes that were displayed sequentially on a highdefinition computer monitor. Each image was classified blindly by the principal investigator in a darkened room. No change in contrast or brightness of these images was undertaken. This evaluation was considered the ground truth.

\subsection{Reproducibility of the method}

To test the reproducibility of the method, a second examiner (C.T.H.) was instructed by the principal observer on the procedure for the obtainment of the ZMS images and on the interpretation of the maturational stages of ZMSs. The second examiner evaluated 30 cases chosen randomly, in which the images were manipulated, the cross sections were selected and the ZMSs were classified, using the same software and classification. After a brief calibration (five cases), this second examiner analysed these cases by herself.

For the definition of the ZMS stages and their identification, three experienced orthodontists (L.F., L.H.S.C., T.N.) who had over 1 year of experience in interpreting CBCT scans for diagnostic purposes in specific research applications were involved in the assessment 
FIGURE 10 Stage B: the ZMS is visualized as a thicker scalloped high density line with some interdigitation at Stage B. In a late Stage B, a thicker scalloped high-density line can be seen in some areas, and in other areas, two thin, parallel, scalloped, high-density lines close to each other and separated by small low-density spaces (arrow) can already be seen at superior (A) and inferior (B) portions. The parasutural bone density still is decreased [Colour figure can be viewed at wileyonlinelibrary.com]
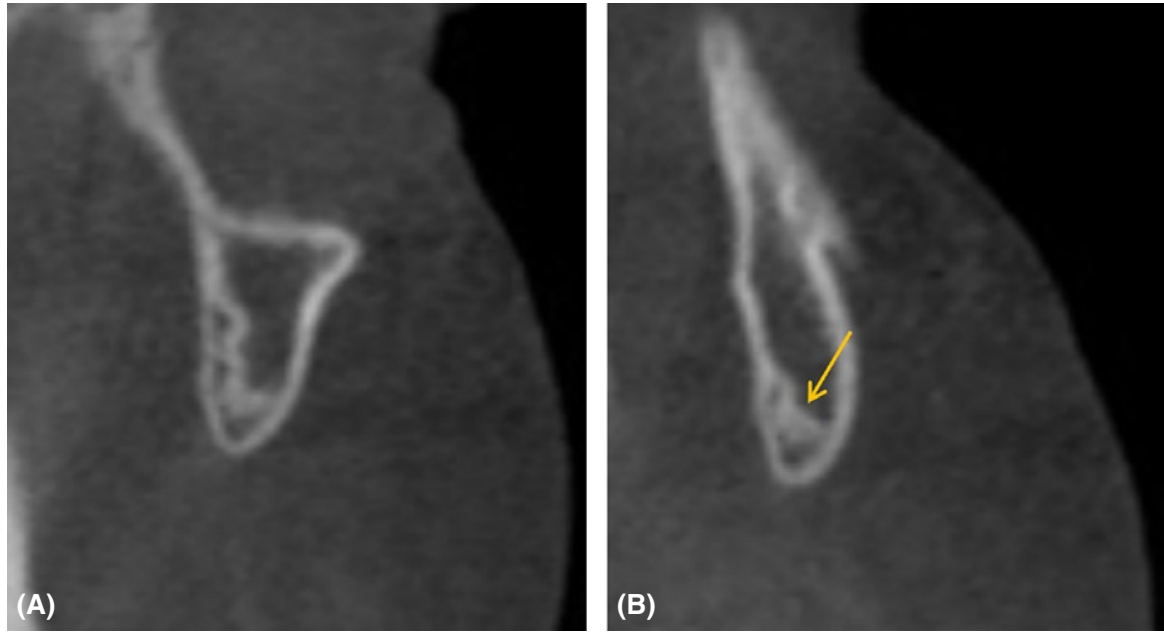

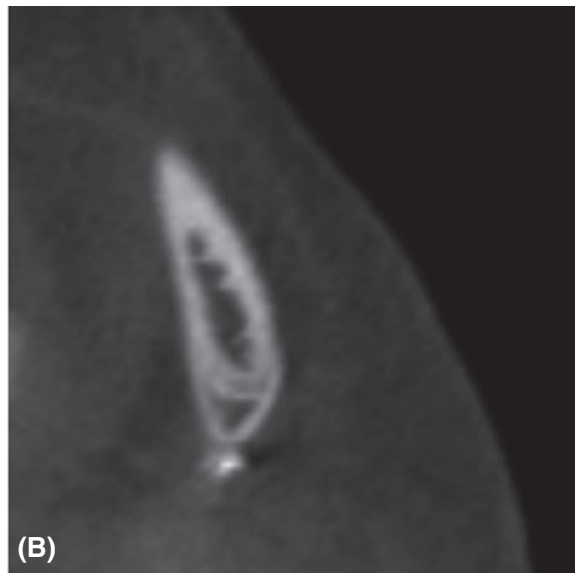

FIGURE 11 Stage C: the ZMS can be seen as two thin, parallel, scalloped, highdensity lines that are close to each other and separated by small low-density spaces in the zygomatic and maxillary bones at superior (A) and inferior portions (B). The parasutural bone density still is decreased

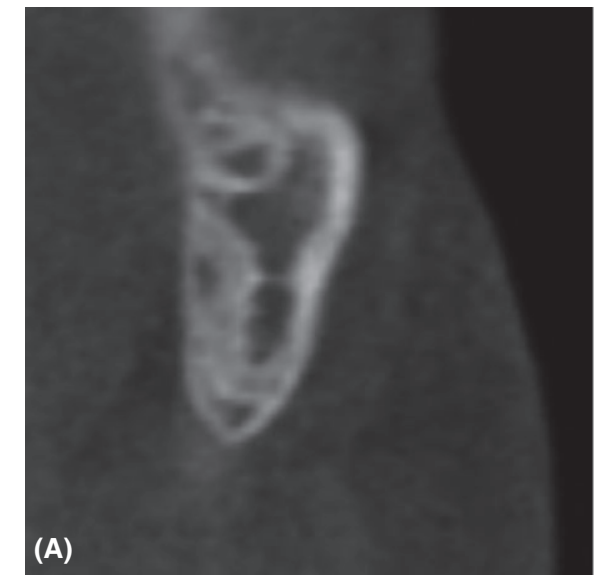

(B)

a PowerPoint presentation with a black background. Examiner calibration was performed using six images, in which all orthodontists openly classified the maturation of ZMSs, and any questions regarding the different maturational stages were discussed.

To test the intra- and inter-examiner reproducibility of the identification of the maturational stages of ZMSs, 20 images were selected randomly to represent all the maturational stages of the ZMS. The three orthodontists classified all images blindly in the same room under dimmed light conditions, using the same high-definition monitor. A second viewing session for the reclassification of the maturation of the ZMS by the same three orthodontists was performed 15 days later in the same way after random rearrangement of the same images.

\section{3 | Statistical analysis}

in a portion of the ZMS, (A), usually in most inferior part (Barrow), where the fusion has occurred. The parasutural bone density is increased in this portion [Colour figure can be viewed at wileyonlinelibrary.com]

of the inter- and intra-observer reproducibility of the identification of the maturational stages of the ZMSs. The definition and figures (Figures 9-13) of the maturational stages of the ZMS were shown on

A weighted kappa coefficient was calculated to evaluate intraexaminer and inter-examiner agreement, as well as the agreement between the examiners and the ground truth. The statistical software used was MedCalc (version 12.3.0; MedCalc Software, Mariakerke, Belgium). The agreement was defined according to the scale of Landis and Koch. ${ }^{27}$ 


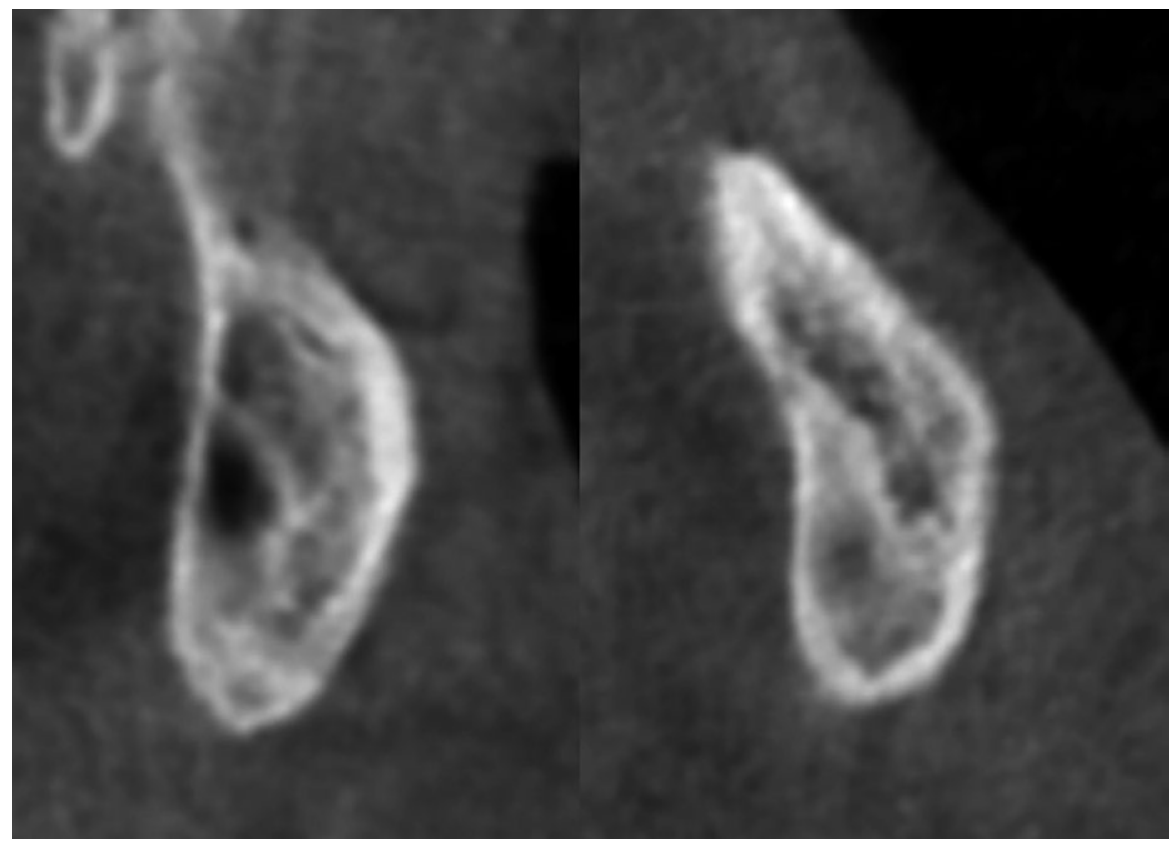

FIGURE 13 Stage E: the ZMS is not visible in many areas along the suture, where fusion of the suture has occurred. The density of the parasutural bone is increased
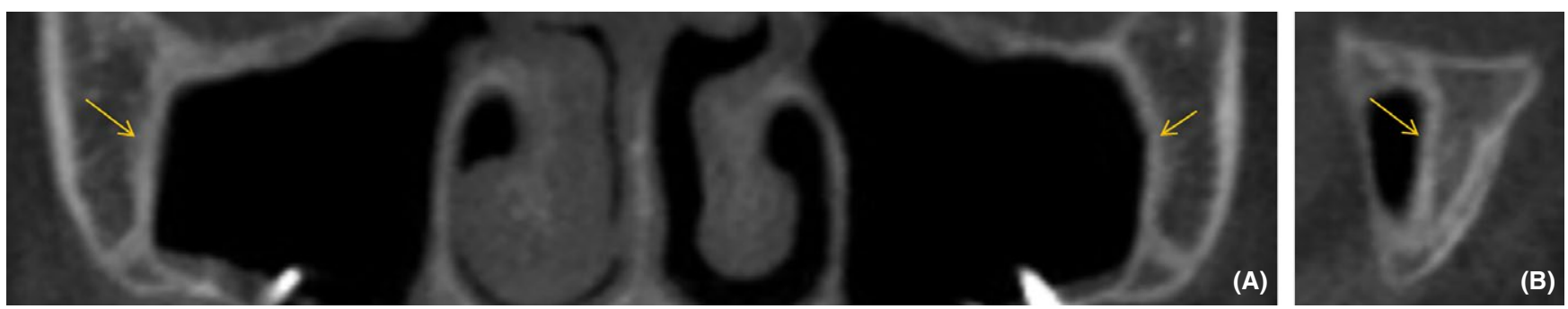

FIGURE 14 For patients who present wide maxillary sinus, the ZMSs can be placed on the lateral wall of the maxillary sinus [Colour figure can be viewed at wileyonlinelibrary.com]

\section{3 | RESULTS}

The method showed a very high inter-examiner reproducibility (weighted kappa coefficient 0.93 with a 95\% confidence interval [Cl] 0.83-1.00).

The weighted kappa values for both intra- and inter-examiner reproducibility of the identification of the maturational stages of ZMSs demonstrated substantial to almost perfect agreement with weighted kappa coefficients ranging from $0.774(95 \% \mathrm{Cl}, 0.560-0.988)$ to 0.953 (95\% Cl, 0.864-1.00]) (Table 2). The reproducibility of the examiners with the ground truth showed almost perfect agreement with weighted kappa coefficients, ranging from $0.855(95 \% \mathrm{Cl}, 0.697$ 1.000 ) to 0.903 (95\% Cl, 0.765-1.00) (Table 2).

The distribution of the maturational stages of ZMS is shown in Table 3. Large variability in the distribution of the maturational stages of ZMS was noted mainly up to 15 years. Stages A, B and C were verified up to 15 years of age, except for one 15.9-year female. Great variability was verified in subjects from 10 to 15 years. No fusion of the ZMS was noted in subjects younger than 10 years old. On the other hand, fusion of ZMS (Stage E) was assessed mainly in subjects older than 15 years.

\section{4 | DISCUSSION}

This study described a new classification for the maturation of the ZMSs. Recently, Angelieri et al. ${ }^{21}$ described a classification of the midpalatal suture maturation on CBCT images. This assessment can be useful mainly for late adolescent and young adult patients to avoid the side effects of RME failure or unnecessary surgically assisted RME. Similarly, the classification for the ZMS maturation could be useful to predict individual responsiveness to maxillary protraction in Class III patients.

The results of the present study showed that the new classification for ZMS maturation is a reproducible method, as both inter- and intraexaminer values demonstrated substantial to almost perfect agreement. However, due to the oblique nature of the ZMSs, the image interpretation protocol used in this study describes the careful standardization of the multiplanar cross-sectional views along the ZMSs. Positioning and visualization of these cross sections may require training and calibration of examiners to avoid errors and inconsistency in choice of anatomic sections to be visualized.

CBCT images analysed in the current investigation were obtained retrospectively, with the spatial resolution ranging from 0.25 to 
TAB LE 2 Weighted Kappa values for inter- and intra-rater agreements and for observers vs ground truth

\begin{tabular}{|lll|}
\hline & Weighted kappa & $\begin{array}{l}95 \% \text { confidence } \\
\text { interval }\end{array}$ \\
\hline Inter rater agreement & & \\
\hline OBS1 vs OBS2 & 0.825 & $0.626-1.000$ \\
\hline OBS1 vs OBS3 & 0.864 & $0.716-1.000$ \\
\hline OBS1 vs OBS4 & 0.774 & $0.560-0.988$ \\
\hline OBS2 vs OBS3 & 0.953 & $0.864-1.000$ \\
\hline OBS2 vs OBS4 & 0.953 & $0.864-1.000$ \\
\hline OBS3 vs OBS4 & 0.903 & $0.775-1.000$ \\
\hline Intra-rater agreement & & \\
\hline OBS1 & 0.742 & $0.589-0.896$ \\
\hline OBS2 & 0.821 & $0.663-0.979$ \\
\hline OBS3 & 0.903 & $0.765-1.000$ \\
\hline OBS4 & 0.758 & $0.565-0.952$ \\
\hline Observers (OBS) vs ground truth (GT) & \\
\hline OBS1 vs GT & 0.774 & $0.630-0.918$ \\
\hline OBS2 vs GT & 0.860 & $0.715-1.000$ \\
\hline OBS3 vs GT & 0.903 & $0.765-1.000$ \\
\hline OBS4 vs GT & 0.855 & $0.697-1.000$ \\
\hline
\end{tabular}

$0.30 \mathrm{~mm}$. According to Waltrick et al., ${ }^{28}$ this variation does not compromise the quality of the images or validity of this study, as voxel sizes of $0.2,0.3 \mathrm{~mm}$ or $0.4 \mathrm{~mm}$ did not influence significant statistically the error of the measurements.

Experimental studies ${ }^{2-5}$ on maxillary protraction have reported that maxillary protraction produced effects on many facial sutures, with sutural response mainly in the transverse palatine suture, zygomaticotemporal sutures and zygomaticomaxillary sutures. The sutural separation was more variable in the ZMSs that showed a greater sutural surface and more complex interdigitation with respect to the other facial sutures. While the transverse palatine suture also may be a possible area of resistance for maxillary protraction, the suture in that area is very thin and does not allow staging of maturation in the current resolution of $\mathrm{CBCT}$ radiographic assessments. Furthermore, Kambara ${ }^{17}$ demonstrated that the ZMSs presented similar histological findings (regarding the maturation), or even greater complexity of interdigitations, compared to other circummaxillary sutures in young (mixed dentition phase) and older (permanent dentition phase) monkeys. Thus, we assume that the other circummaxillary sutures probably will present the same or less matured maturational stages compared to the ZMSs.

Interestingly, the ZMS is well visualized on CBCT images due to its robust thickness, in contrast to the zygomaticotemporal and transverse palatine sutures. Its complexity of shape and tortuousness ${ }^{22}$ requires that two slices should be evaluated at the infraorbital (superior) and infrazygomatic (inferior) portions of the suture.

The radiographic examination of the ZMS revealed similar maturational stages compared to the maturation of the midpalatal suture. ${ }^{21}$ Despite the tortuous shape of the ZMS, low density of parasutural bone at less mature stages and the increase in interdigitation of suture as maturation progresses also were noted. The presence of the two lines as two thin, parallel, scalloped, high-density lines close to each other and separated by small low-density spaces (stage C) also preceded the fusion of the ZMS. These findings corroborate those of Cohen $^{24}$ who demonstrated that facial sutures have similar maturation processes. Obviously, both ZMSs should be examined, because for some patients, two different stages were identified bilaterally. For those patients, the ZMS staging was considered as the more matured side.

As for the distribution of the developmental stages of the ZMS (Table 3), no fusion of ZMS was observed in subjects younger than 10 years, while fusion of ZMS (stages $D$ and $E$ ) was assessed mainly in subjects older than 15 years. It is interesting to note that between 10 and 15 years, there is great variability of ZMS maturational stages in that all stages can be found during this age interval.

The individual assessment of the circummaxillary suture maturation may provide a means of assessing the midfacial sutural response to applied orthopedic forces in Class III patients. On the basis of the present results, we can speculate that at stages $A$ and $B$, a conventional treatment approach for Class III malocclusion, like the RME and facial mask protocol, would encounter less resistant forces to maxillary protraction. At stages A and B, presumably, greater skeletal effects associated with maxillary protraction can be expected than at stage $\mathrm{C}$, when there are initial ossification areas along the ZMSs.

In the presence of stages $D$ and $E$ that show partial or complete fusion, dentoalveolar effects have to be expected as the primary result of maxillary protraction. If maxillary advancement is required at these more mature stages, a surgical approach should be considered. Due to the great variability in ZMS maturational stages between 10
TABLE 3 Distribution of the maturational stages of the ZMS

\begin{tabular}{|c|c|c|c|c|c|c|c|}
\hline \multirow[b]{2}{*}{ Stages } & \multicolumn{2}{|l|}{$5-<10 y$} & \multicolumn{2}{|c|}{$10-<15 y$} & \multicolumn{2}{|l|}{$>15 y$} & \multirow[b]{2}{*}{ Total } \\
\hline & Female & Male & Female & Male & Female & Male & \\
\hline A & 6 & 1 & 1 & 2 & 0 & 0 & 10 \\
\hline B & 7 & 0 & 7 & 5 & 0 & 0 & 19 \\
\hline C & 4 & 2 & 4 & 4 & 1 & 0 & 15 \\
\hline D & 0 & 0 & 2 & 2 & 4 & 0 & 8 \\
\hline$E$ & 0 & 0 & 1 & 1 & 13 & 7 & 22 \\
\hline Total & 17 & 3 & 15 & 14 & 18 & 7 & 74 \\
\hline
\end{tabular}


and 15 years, the evaluation ZMS maturational stages appears to be indicated mainly in juvenile and early adolescent Class III patients. However, future studies with the larger sample are indicated for the clinical meaning of the different ZMS stages before the clinical applications of this classification method.

\section{5 | CONCLUSIONS}

The classification of ZMS maturation using CBCT appears to be a reliable method that allows the assessment of the morphology of the ZMSs in the individual patient. This method could be useful to predict individual patient responsiveness to rapid maxillary expansion and maxillary protraction. Nevertheless, future studies are recommended evaluating the clinical meaning of the different ZMSs stages before the clinical application of this method.

\section{REFERENCES}

1. Melsen B, Melsen F. The postnatal development of the palatomaxillary region studied on human autopsy material. Am J Orthod. 1982;82:329-342.

2. Kambara T. Dentofacial changes produced by extraoral forward force in the Macaca irus. Am J Orthod. 1977;71:249-277.

3. Jackson G, Kokich V, Shapiro P. Experimental and post-experimental response to anteriorly directed extraoral force in young Macaca nemestrina. Am J Orthod. 1979;75:318-333.

4. Nanda R, Hickory W. Zygomaticomaxillary suture adaptations incident to anteriorly-directed forces in rhesus monkeys. Angle Orthod. 1984;54:199-210.

5. Zhao N, Xu Y, Chen Y, Xu Y, Han X, Wang L. Effects of class III magnetic orthopedic forces on the craniofacial sutures of rhesus monkeys. Am J Orthod Dentofacial Orthop. 2008;133:401-409.

6. Kapust AJ, Sinclair PM, Turley PK. Cephalometric effects of facemask/ expansion therapy in class III children: a comparison of three age groups. Am J Orthod Dentofacial Orthop. 1998;113:204-212.

7. Franchi L, Baccetti T, McNamara JA Jr. Postpubertal assessment of treatment timing for maxillary expansion and protraction therapy followed by fixed appliances. Am J Orthod Dentofacial Orthop. 2004;126:555-568.

8. Baccetti T, Franchi L, McNamara JA Jr. Treatment and posttreatment craniofacial changes after rapid maxillary expansion and facemask therapy. Am J Orthod Dentofacial Orthop. 2000;118:404-413.

9. Cordasco G, Matarese G, Rustico L, et al. Efficacy of orthopedic treatment with protraction facemask on skeletal class III malocclusion: a systematic review and meta-analysis. Orthod Craniofac Res. 2014;17:133-143.

10. Macdonald KE, Kapust AJ, Turley PK. Cephalometric changes after the correction of class III malocclusion with maxillary expansion/facemask therapy. Am J Orthod Dentofacial Orthop. 1999;116:13-24.

11. Ngan P, Yiu C, Hu A, Hägg U, Wei SH, Gunel E. Cephalometric and occlusal changes following maxillary expansion and protraction. Eur J Orthod. 1998;20:237-254.
12. Hickham JH. Maxillary protraction therapy: diagnosis and treatment. J Clin Orthod. 1991;25:102-113.

13. Proffit WR, ed. Contemporary Orthodontics. St Louis: CV Mosby; 1992.

14. Wells AP, Sarver DM, Proffit WR. Long-term efficacy of reverse pull headgear therapy. Angle Orthod. 2006;76:915-922.

15. Yüksel S, Uçem TT, Keykubat A. Early and late facemask therapy. Eur J Orthod. 2001;23:559-568.

16. Yavuz I, Halicioğlu K, Ceylan I. Face mask therapy effects in two skeletal maturation groups of female subjects with skeletal class III malocclusions. Angle Orthod. 2009;79:842-848.

17. Kambara T. Dentofacial changes produced by extraoral forward force in the Macaca irus. Am J Orthod. 1977;71:249-277.

18. Yu HS, Baik HS, Sung SJ, Kim KD, Cho YS. Three-dimensional finiteelement analysis of maxillary protraction with and without rapid palatal expansion. Eur J Orthod. 2007;29:118-125.

19. Tanne K, Sakuda M. Biomechanical and clinical changes of the craniofacial complex from orthopedic maxillary protraction. Angle Orthod. 1991;61:145-152.

20. De Vos W, Casselman J, Swennen GR. Cone-beam computerized tomography (CBCT) imaging of the oral and maxillofacial region: a systematic review of the literature. Int J Oral Maxillofac Surg. 2009;38:609-625.

21. Angelieri F, Cevidanes LHS, Franchi L, Gonçalves JR, Benavides E, McNamara JA Jr. Midpalatal suture maturation: classification method for individual assessment prior to rapid maxillary expansion. Am J Orthod Dentofacial Orthop. 2013;144:759-769.

22. Sholts SB, Wärmländer SKTS. Zygomaticomaxillary suture shape analyzed with digital morphometrics: reassessing patterns of variation in American Indian and European populations. Forensic Sci Int. 2012;217:234e.1-234e.6.

23. Sun Z, Lee E, Herring SW. Cranial sutures and bones: growth and fusion in relation to masticatory strain. Anat Rec A Discov Mol Cell Evol Biol. 2004;276:1-22.

24. Cohen MM Jr. Sutural biology and the correlates of craniosynostosis. Am J Med Gen. 1993;47:581-616.

25. Hahn W, Fricke-Zech S, Fialka-Fricke J, et al. Imaging of the midpalatal suture in a porcine model: flat-panel volume computed tomography compared with multislice computed tomography. Oral Surg Oral Med Oral Pathol Oral Radiol Endod. 2009;108:443-449.

26. Korbmacher H, Schilling A, Püschel K, Amling M, Kahl-Nieke B. Agedependent three-dimensional micro-computed tomography analysis of the human midpalatal suture. J Orofacial Orthop. 2007;68:364-376.

27. Landis JR, Koch GG. The measurement of observer agreement for categorical data. Biometrics. 1977;33:159-174.

28. Waltrick KB, Nunes de Abreu Junior MJ, Corrêa M, Zastrow MD, Dutra VD. Accuracy of linear measurements and visibility of the mandibular canal of cone-beam computed tomography images with different voxel sizes: an in vitro study. J Periodontol. 2013;84:68-77.

How to cite this article: Angelieri F, Franchi L, Cevidanes LHS, Hino CT, Nguyen T, McNamara Jr JA. Zygomaticomaxillary suture maturation: A predictor of maxillary protraction? Part I - A classification method. Orthod Craniofac Res. 2017;20:85-94. https://doi.org/10.1111/ocr.12143 